\title{
Skin Recovery After Discontinuation of Long-Term Moisturizer Application: A Split-Face Comparison Pilot Study
}

\author{
Julia-Tatjana Maul · Lara Valeska Maul • Marc Kägi · Phil Cheng • \\ Florian Anzengruber · Mathilde von Laue • Yuki Chen · \\ Martin Kägi · Alexander Navarini
}

Received: August 12, 2020 / Published online: October 7, 2020

(C) The Author(s) 2020

\begin{abstract}
Introduction: Facial moisturizers are commonly used by healthy women and increasingly men of all age groups. This study aimed to investigate the effects of moisturizer discontinuation and the subsequent evolution of symptoms.

Methods: Two prospective observational splitface comparison pilot studies were performed in

Julia-Tatjana Maul and Lara Valeska Maul contributed equally to this work.
\end{abstract}

Electronic supplementary material The online version of this article (https://doi.org/10.1007/s13555020-00453-0) contains supplementary material, which is available to authorized users.

J.-T. Maul $(\bowtie) \cdot$ P. Cheng · F. Anzengruber

Department of Dermatology, University Hospital of Zürich, Zurich, Switzerland

e-mail: Julia-Tatjana.Maul@usz.ch

L. V. Maul · A. Navarini

Department of Dermatology, University Hospital of Basel, Basel, Switzerland

M. Kägi · M. von Laue · M. Kägi

Faculty of Medicine, University of Zürich, Zurich, Switzerland

Y. Chen

Faculty of Medicine, University of Bern, Bern, Switzerland

M. Kägi · M. Kägi

Hautzentrum, Zürich AG, Zurich, Switzerland
Switzerland and enrolled (I) 20 healthy women aged 17-25 years in winter and (II) 36 female subjects $15-20$ and $40-55$ years of age in summer. Moisturizers were stopped on the investigational half of the face. On the control side, the usual skin care regimen was continued. Daily subjective (I/II) and objective (I) skin assessments for the occurrence of typical symptoms of dry skin (dryness, itching, scales, redness, wrinkles) were collected.

Results: In the winter study (cohort I) in both the subjective and objective assessment, all skin changes increased significantly within 1 day after discontinuation. On day 7 , dryness $(p<0.001)$, itching $(p<0.025)$, redness $(p<0.001)$ and scales $(p<0.049)$ were significantly different in the subjective assessment and redness $(p<0.004)$ and scales $(p<0.001)$ in the objective assessment. Skin dryness reverted to baseline levels after 6 days in the objective assessment and 10 days in the subjective assessment. The control side's condition was reached after 6 days. In the summer study (II), only among the 15-20-year-olds was dryness significantly higher on the intervention side from day $1 \quad(p<0.028)$ to day $14 \quad(p<0.009)$. Their recovery time was 11 days until dryness intensity scores comparable to baseline were reached, and 21 days until the control side's values were matched. Over a 7-day period, the overall mean dryness score was significantly different between the interventional and control sides for both young and old participants. 
Conclusions: Both healthy young and aging female subjects react with typical symptoms of temporary dryness to a sudden stop of a previous long-term moisturizer treatment but regain normal levels quickly without continuation of moisturizers. The skin recovery time for skin dehydration is $1-3$ weeks in young female subjects with varying intensities depending on the season.

Keywords: Discontinuation; Dryness; Facial moisturizer; Long-term use; Skin

\section{Key Summary Points}

Facial moisturizers are commonly used by healthy women and increasingly men of all age groups.

The effects of moisturizer discontinuation and the skin recovery time after subsequent symptoms need to be investigated.

The hypothesis was that the skin of female subjects of any age will recover within 1 month.

Regardless of the season, both healthy young and aging female subjects react with typical symptoms of temporary dryness to a sudden stop of previous longterm moisturizer use.

The skin hydration of young female subjects recovers after moisturizer discontinuation in less than a month.

\section{DIGITAL FEATURES}

This article is published with digital features to facilitate understanding of the article. You can access the digital features on the article's associated Figshare page. To view digital features for this article go to https://doi.org/10.6084/m9. figshare.12967268.

\section{INTRODUCTION}

Dry skin commonly affects people of all skin types and can occur on various areas of the body. Xerosis is triggered by both endogenous factors e.g. hormone fluctuations, diseases (atopic disease, psoriasis, thyroiditis) and medication (retinoids) and exogenous factors e.g. environment, climate [1]. Dryness of the skin is characterized by skin thickening or thinning, by roughness, reddening, scales, irritation and itching [2]. In addition, fine wrinkles are also a typical sign of dehydrated skin.

The physiological mechanism of skin moisturization consists of a stratum corneum (SC) containing $10-15 \%$ water in order to remain flexible and elastic. The SC comprises predominantly hydrophobic substances and is an essential factor that slows down corneocyte dehydration and ensures enzymatic activity [1]. Dry skin is characterized by a moisture content of less than $10 \%$, which is coherent with a loss of continuity of the SC [1]. Transepidermal water loss (TEWL) is the most widely used surrogate marker for skin barrier function [3].

Moisturizers enhance the water-holding capacity of the skin. They are commonly used by all age groups and in both genders regardless of the presence of a skin disease. Functional skin benefits are sought with application of these products on facial skin by making the skin soft and smoother, increasing skin hydration and skin rejuvenation. Indeed, the application of moisturizers achieves an appearance benefit [4]. However, recent studies suggest that long-term therapy with moisturizers not only strengthens but also weakens the skin barrier function and may influence its recovery subsequent to damage [5-7].

The aim of this prospective observational study was to determine the effect of moisturizer discontinuation and the temporal evolution of subsequent symptoms. Five variables were determined to indicate the occurrence of dry facial skin in this study: dryness, itching, scales, redness and wrinkles. Furthermore, this study investigates whether the skin recovers to the original condition and, if so, how long this recovery process takes. 
The null hypothesis of our study is that sudden discontinuation of daily moisturizer application in female subjects leads to longterm skin discomfort.

\section{METHODS}

\section{Participating Population and Data Collection}

Female participants $(n=56)$ between 15 and 55 years of age who used moisturizers on a regular basis were prospectively included in this pilot study consisting of a winter cohort I and summer cohort II. The two prospective observational split-face comparison studies were performed in Switzerland in winter 2016/2017 and summer 2017 to investigate the impact of a sudden discontinuation of daily long-term moisturizer use on facial skin. The inclusion criteria consisted of female participants who were long-term users of moisturizing cream for at least 2 weeks in cohort I and for at least 4 weeks in cohort II and did not have any skin diseases requiring medical therapy. Other topical treatments besides water, make-up and make-up remover were not allowed.

The 2-week study (cohort I) included 20 women aged 17-25 years during December 2016 until February 2017 in Zürich, Switzerland. Performing a half-side test, the subjects stopped using their usual moisturizer on the right side of their face, which was considered the intervention side. The usual skin care with the participants' own standardized moisturizer was continued on the left side of the face, the control side, without any change in frequency or amount applied. Every participant received written instructions and information about skin care to prevent obvious misuse. On the basis of a daily assessment sheet, the condition of the skin on both halves of the face was recorded by the participants themselves according to the subjective perception at baseline and at days 1-14. The assessment was based on five symptoms: dryness, itching, scales, reddening and wrinkles. The questionnaire was completed every evening before water contact on a visual analogue scale (VAS) of 1-10 for each symptom whereby 1 represented an absence of the symptom and 10 a severe occurrence of the symptom [8]. Subjective dryness was perceived by participants touching their own skin and judging suppleness and flexibility. Objective dryness was measured by visual identification of reduced reflection due to lacking lipids. Scales were visually identified owing to the presence of whitish exfoliating elements of the SC. Additional information about the participants' skin care habits and their attitude towards the examination were recorded.

In addition to the subjective VAS, an objective assessment was conducted with standardized digital photographs. The photos were taken by all 20 participants themselves using a mobile phone camera with a resolution of at least 1.2 megapixels in the selfie mode with a predefined distance of $30 \mathrm{~cm}$ from face to bathroom mirror. Overall 20 pictures were taken every evening by the participants, without wearing make-up, in a well-lit room with a light source symmetrically illuminating the face of the subject. The patients did not consent to the publication of their photos.

The summer study (II), a 3-week split-face comparison, prospective observational study, was performed in Aarau, Switzerland from May to September 2017 with 36 female subjects aged 15-20 and 40-55 years. The aim was to determine whether age and/or seasons have an impact on facial skin changes after sudden removal of the usual moisturizing cream. A younger age group (15-20 years) with 23 female subjects was compared to an older age group (40-55 years) with 13 women. The 36 self-selected volunteer participants agreed to perform the aforementioned study with daily clinical subjective evaluations. No objective data analysis was accomplished because the quality of the photos taken was insufficient. The left facial side was the intervention side, while the right facial side was the control side.

Two different study durations were chosen because of the season-dependent moisture behaviour of the skin. 


\section{Statistical Analysis}

Descriptive analyses were performed using standard parameters [means, standard deviation (SD)] for each symptom. Dryness score was calculated by taking the average of dryness, itching, scales, redness and wrinkles. Data points were plotted over time and smoothened with LOESS regression. For statistical analysis, the Student's $t$ test was used to compare means. A $p$ value less than 0.05 was deemed significant. Missing data were not imputed.

The two studies were performed as college theses with healthy persons and were exempt from ethical review, which was ascertained by consultation with the Swiss Ethics Committee prior to the start of the study (Ethics Committee Northwest Switzerland EKNZ 2020-00667). The studies were performed in full accordance with the Declaration of Helsinki (1964) and its subsequent amendments. Good Clinical Practice was maintained throughout the studies. Informed consent was received from the patients for inclusion in the study.

\section{RESULTS}

According to weather records from Meteo Switzerland in winter 2016/2017, the average temperature was $0.4{ }^{\circ} \mathrm{C}$, the average precipitation was $221 \mathrm{~mm}$ and the average sunshine duration was $142 \mathrm{~h}$ in Zürich [9].

\section{Winter Study (Cohort I)}

In the first analysis, 20 women between 17 and 25 years performed a daily photo documentation over 2 weeks in addition to their selfassessments (supplementary Table 1; Fig. 1).

Within the first 7 days of stopping facial moisturizing cream, the mean skin dryness score on the interventional facial side significantly increased in both the subjective (4.37 vs $2.62, p<0.001)$ and objective $(3.78$ vs 2.75 , $p<0.001)$ assessment compared to the control facial side (Table 1; Fig. 1).

Whereas at days 8-14, no significant differences were observed in both the subjective $(p<0.084)$ and the objective assessment $(p<0.563)$ for skin dryness (data not shown). The skin condition of the investigated facial side was at the same state as the control side after 6 days in the objective assessment and after 10 days in the subjective assessment (Fig. 2).

The skin recovery time is defined as time until the skin reaches its condition at baseline. This condition was achieved of the investigated side after 6 days in the objective assessment and after 10 days in the subjective assessment (Fig. 2).

A significant increase in all analysed skin changes was observed at day 1 in the subjective assessments for dryness, scales, itching, redness and wrinkles $(p<0.001)$. On day 7 , only dryness $(p<0.001)$, itching $(p<0.025)$, redness $(p<0.001)$ and scales $(p<0.049)$ were significantly different. On day 14 , however, the differences had disappeared again except for itching $(p<0.047)$, which was higher in the control side compared to the intervention side (supplementary Fig. 2 right graphs, Table 2A). In addition, in the objective analysis at day 1 , all observed skin changes increased significantly: dryness and scales $(p<0.001)$, itching $(p<0.010)$, redness $(p<0.015)$ and wrinkles $(p<0.003)$. Interestingly, on day 7 , dryness $(p<0.001)$ was significantly higher in the control side, whereas redness $(p<0.004)$ and scales $(p<0.001)$ were rated significantly higher on the intervention side. By day 14, differences had disappeared (supplementary Fig. 2 left graphs, Table 2B).

Besides an increased subjective perception of skin dryness, the course of the skin changes was similar in the objective and subjective analyses with a high fluctuation of all mean symptom values and an adjustment to the original condition in both subgroups over the study observation period.

\section{Summer Study (Cohort II)}

According to weather records from Meteo Switzerland in summer 2017, the average temperature was $19.5^{\circ} \mathrm{C}$, the average precipitation 

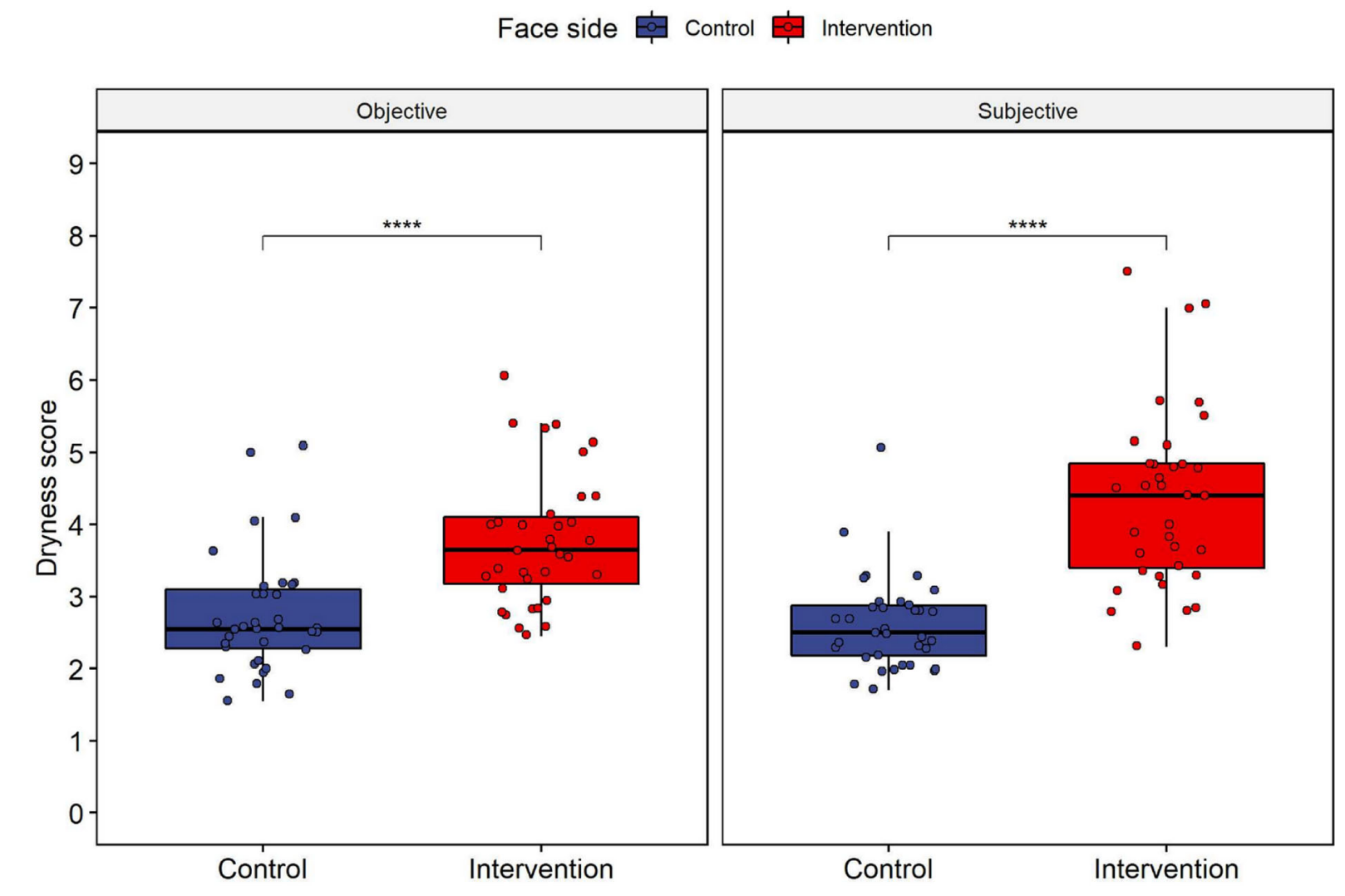

Fig. 1 Winter study: boxplots comparing the mean dryness score (mean of dryness, itching, redness, scales and wrinkles) from days 1 to 7 between the objective and

subjective evaluation of control and intervention face sides. Student's $t$ test was performed to compare means: ${ }^{* * * *} p<0.0001$

In the young age group, 15-20-year-old Table 1 Winter study: mean dryness score (mean of dryness, itching, redness, scales and wrinkles) from day 1 to 7 of objective and subjective evaluation of control and intervention face sides

\begin{tabular}{llll}
\hline & Control & Intervention & $\boldsymbol{p}_{\text {value }}$ \\
\hline Objective & $2.75(0.83)$ & $3.78(0.91)$ & $<0.0001$ \\
Subjective & $2.62(0.65)$ & $4.37(1.24)$ & $<0.0001$ \\
\hline
\end{tabular}

Data presented as mean (SD)

a From Student's $t$ test

was $360 \mathrm{~mm}$ and the average sunshine duration was $679 \mathrm{~h}$ in Zürich [10].

In the second study, 36 female participants completed the 3-week split-face comparison study (supplementary Table 1, Fig. 1). subjects, an increased mean dryness score during days 1-7 was detected on the intervention side compared to the control facial side $(p<0.0001)$ (supplementary Table 3; Fig. 4). The immediate significant increase of the perception of skin dryness on the intervention side of the face at day $1(p<0.027)$ was reported after discontinuing the moisturizing cream. Over a 7-day period, the overall mean dryness score was significantly higher between the interventional and control sides for both young and old participants, albeit the difference was smaller in the older group (Table 2; Fig. 3). In the old age group, the intervention side never recovered to the original condition after 21 days, whereas in the young age group the interventional side reached the same state as the control side after 21 days. If we analyse the time until the skin reaches its condition at 


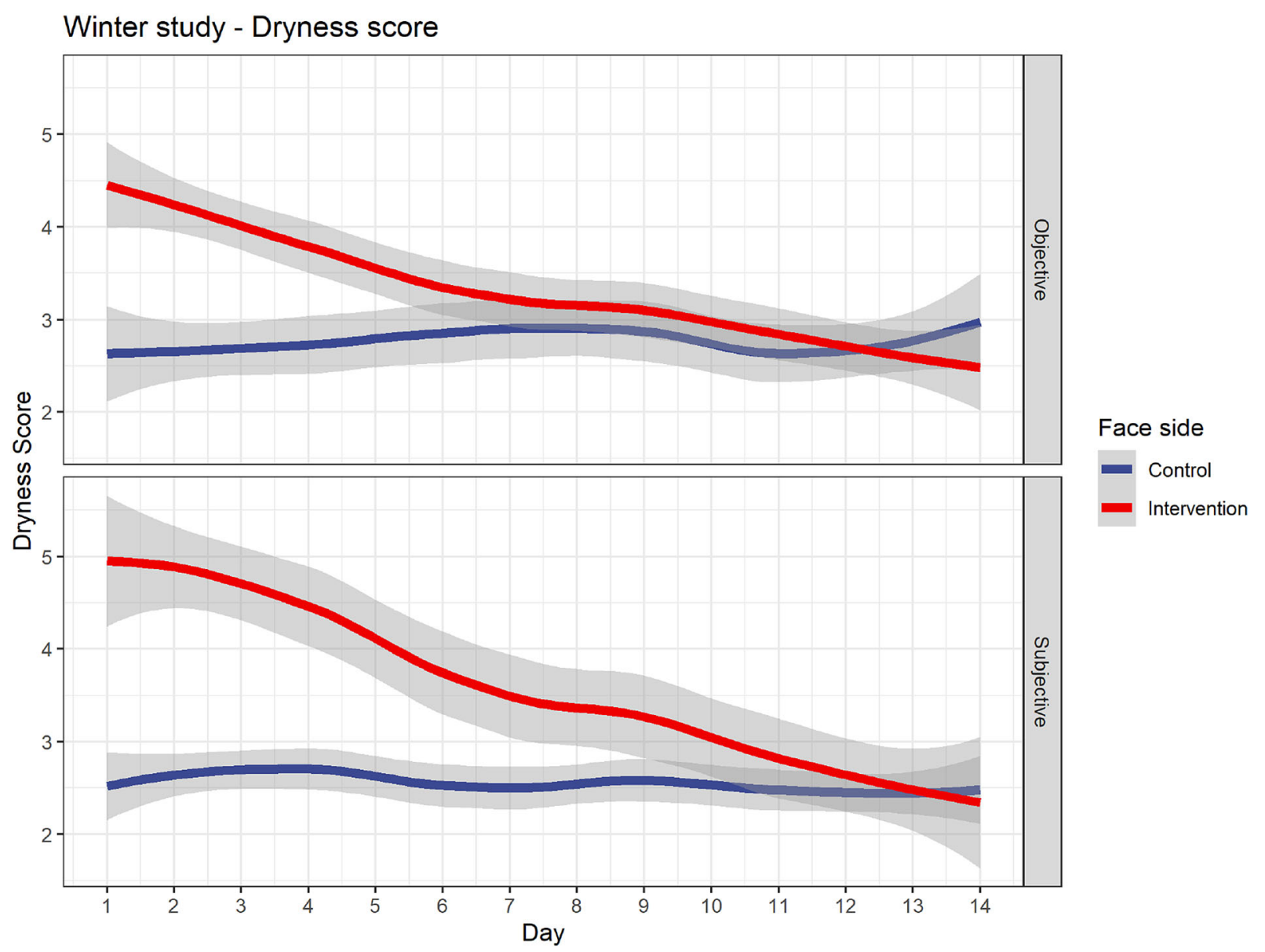

Fig. 2 Winter study: timeline of dryness score (mean of dryness, itching, redness, scales and wrinkles) from day 1 to 14 comparing control (blue) and intervention (red) face

Table 2 Summer study: mean dryness score (mean of dryness, itching, redness, scales and wrinkles) from day 1 to 7 of old and young groups comparing control and intervention face sides

\begin{tabular}{llll}
\hline & Control & Intervention & $\boldsymbol{p}$ value $^{\mathbf{a}}$ \\
\hline Old & $1.79(1.66)$ & $1.97(1.73)$ & 0.0022 \\
Young & $1.69(1.14)$ & $2.02(1.44)$ & $<0.0001$ \\
\hline
\end{tabular}

Data are presented as mean $(\mathrm{SD})$

${ }^{a}$ From Student's $t$ test

baseline, the young age group showed a recovery time for dryness of 11 days (Fig. 4). sides for objective and subjective evaluations. Line was smoothened using LOESS regression and shaded area is the 95\% confidence interval

Among the 15-20-year-old subjects, there was no significant difference in the development of scales, itching, reddening and wrinkles between interventional and control facial side. Only dryness was significantly different between the intervention and control side from day 1 to 14 (supplementary Table 3A; Fig. 3 right side graphs).

In the older age group, 40-55-year-old subjects, there were no significant differences between the interventional and control side in the subjects' self-assessment in any of the skin parameters throughout 21 days (supplementary Fig. 3-left side graphs, Table 3B). 
Face side 투 ontrol 투 Intervention
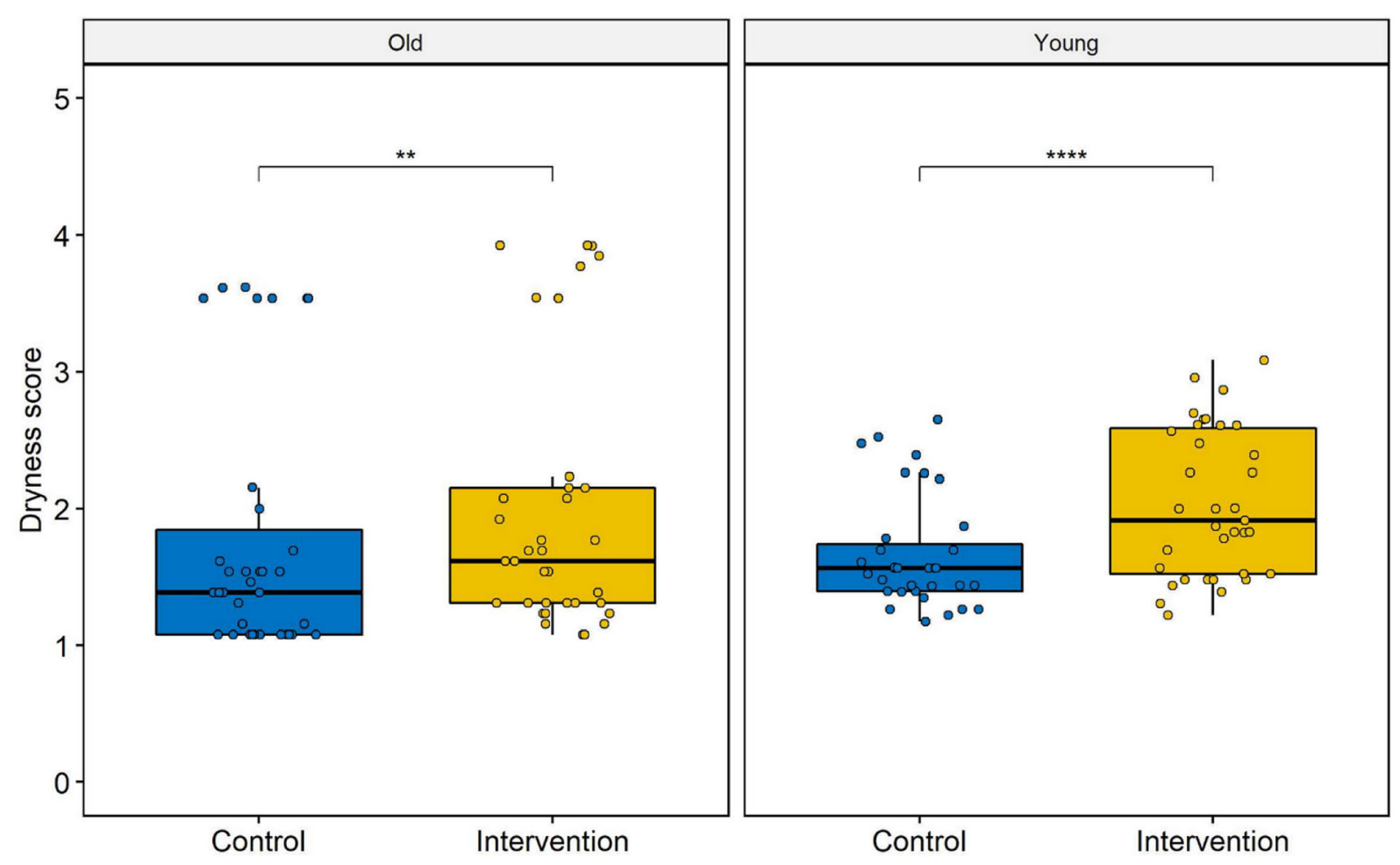

Fig. 3 Summer study: boxplots comparing the mean dryness score (mean of dryness, itching, redness, scales and wrinkles) from day 1 to 7 between the old and young

group evaluation of control and intervention face sides. Student's $t$ test was performed to compare means: ${ }^{* *} p<0.01 ;{ }^{* * * *} p<0.0001$

\section{Narrative Data Concerning Participants' Doubts}

Additional personal messages from the participants of the summer study (cohort II) showed that stopping use of moisturizers is a big challenge for female subjects in general. Depending on the age group, distinct worries were voiced. At the beginning of the clinical study, the older women showed worries concerning the possible appearance of wrinkles. They did not seem to worry about possible redness, scales or itching. The concerns of the younger group of subjects, however, were mainly about itching and scales. None of the young participants mentioned any fear of possible wrinkles. All older women started their moisturising treatment again after completion of the study (supplementary Table 4).

\section{DISCUSSION}

Our analysis of two independent cohorts demonstrates that a sudden discontinuation of daily moisturizing creams leads to significant but temporary symptoms that suggest relevant dehydration of the facial skin. In the cohort I of 17-25-year-old women during winter, typical symptoms of dry skin already appeared on day 1 after treatment discontinuation. Both subjective and objective assessments confirmed the observed significantly increased effects. Additionally, in summer (cohort II), dryness of facial skin was significantly higher only in the 15-20year-old participants.

To the best of our knowledge, this is the first clinical study analysing whether discontinuation of facial moisturizing cream leads to skin changes in the real-world setting. The aim was 


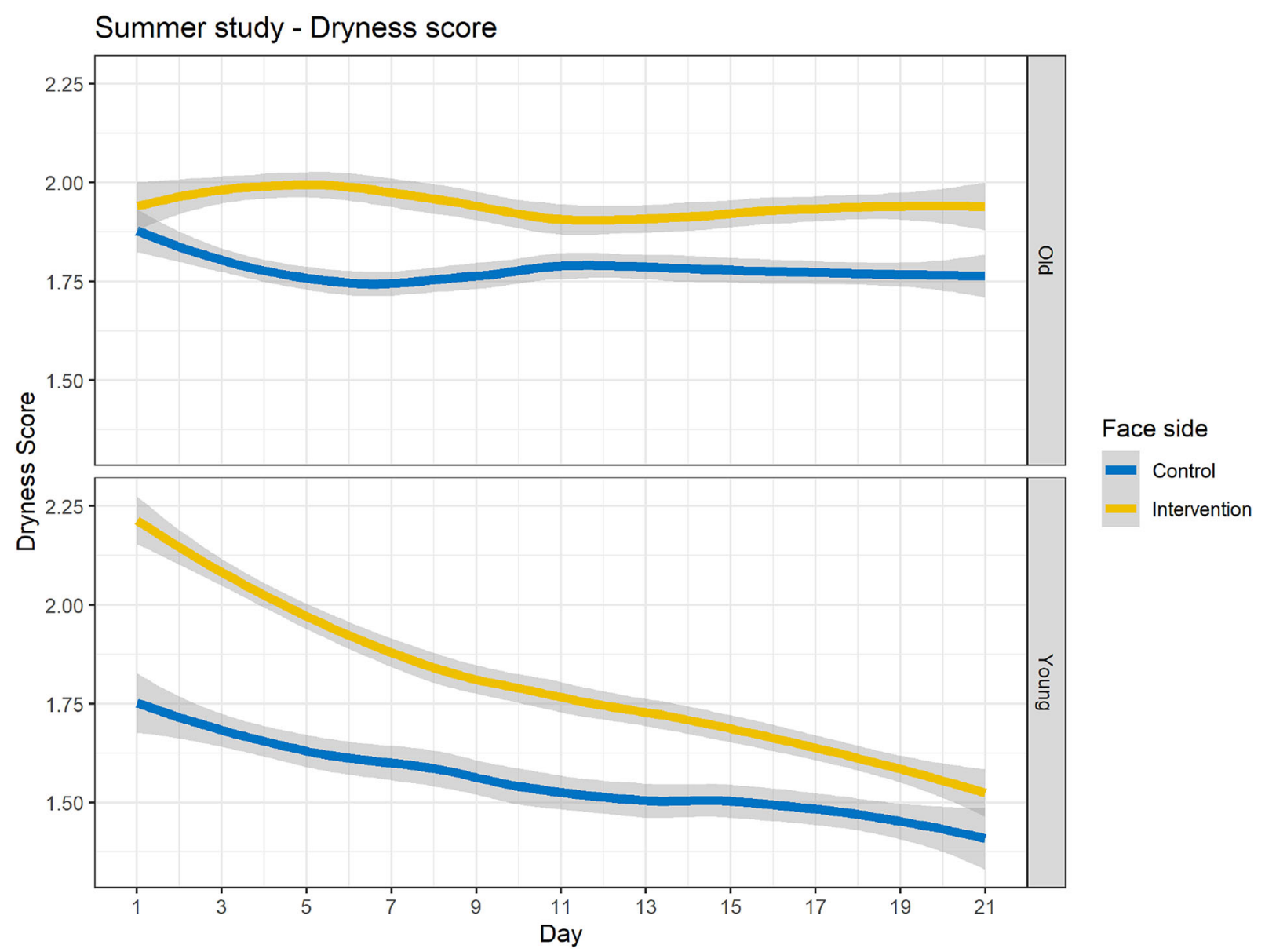

Fig. 4 Summer study: timeline of dryness score (mean of dryness, itching, redness, scales and wrinkles) from day 1 to 14 comparing control (blue) and intervention (yellow) face

to evaluate whether the skin recovers to its original condition and, if so, how long this recovery process takes.

Knowledge about benefits and disadvantages of long-term therapy with moisturizers influencing the skin barrier function and the skin's reaction to a sudden stop of this treatment is still insufficient [1]. A standard facial moisturizer consists of approximately $80 \%$ water, $5 \%$ humectants, $4 \%$ emollients/occlusives, $6 \%$ emulsifiers, $2 \%$ silicate, $0.3 \%$ thickener, $0.4 \%$ preservative, and $0.2 \%$ fragrance. Topical moisturizers are able to deposit lipids and other permeability barrier-enhancing ingredients within the SC and provide occlusion causing a more immediate reduction in TEWL [11]. sides for the old and young groups. Line was smoothened using LOESS regression and shaded area is the 95\% confidence interval

Natural moisturizing factor, a collection of humectant substances based on the catabolism of filaggrin, serves as a successful component in moisturizers [12]. By inducing changes in the superficial and deep layers of the epidermis, the use of over-the-counter (OTC) moisturizers may have multiple beneficial, but also potentially negative effects on the skin $[5,7,13,14]$. Improvements in barrier function of the SC with topical moisturizers measured by TEWL and confocal Raman spectroscopy have frequently been reported $[7,15,16]$. An increased TEWL occurs under lipid-free gel, whereas adding lipids results in an increased effectiveness regarding skin dryness [17]. Long-term moisturizing treatment may lead to reduced baseline 
sebum levels in Caucasians, which makes the skin more vulnerable including increased skin susceptibility to irritants [18]. Consistent with our results, regardless of the season, a previous study has shown an immediate decreasing hydration score after stopping moisturizer application [19].

Various exogenous factors such as climate, seasons and extreme bathing habits have been proposed to be linked to dry skin [20, 21]. Despite external challenges, the renewal process can mostly compensate to maintain the skin barrier's protective function [12]. In accordance with our data, we noticed only minor seasonal differences in skin recovery time. An essential part of the homeostatic function of the SC in every individual is the maintenance of skin hydration regardless of the presence of skin disease or normal skin [22]. In contrast, seasonal variation of catalase activity in human SC, with lower activities in summer and higher activities in winter, suggest seasonal differences. Hellemans et al. detected that sun exposure results in disturbed antioxidant enzyme activity leading to increased vulnerability to oxidative damage in the SC barrier components with low activities in summer and higher activities in winter [22]. They indicate that the recovery of the catalase activity shows seasonal variation with an agedependent recovery being less in younger adults compared to older adults.

Former studies showed that TEWL and consequently the visual dryness are significantly higher in winter than in summer. The SC lipids (total and individual ceramides, total fatty acids and cholesterol) are higher in summer compared to winter. Further seasonal functional changes of the skin barrier include significant higher indicators of skin inflammation, the ratio of interleukin-1 receptor antagonist (IL1ra) to interleukin-1 $\alpha$ (IL-1 $\alpha)$, in the winter [23]. We assume that healthy skin's greater need for moisturizing in winter explains why the facial dryness was more intense in our winter study.

Older persons' skin reacts less intensely than young persons' skin regarding changes caused by interruption of topical moisturizer. Except for the significantly increased overall mean dryness score in aging skin over a 7-day period, there were no further significant differences between the interventional and control sides in older participants. We conclude that the decreased lipid and intrinsic moisturization of aged skin might be less quickly downregulated during treatment with moisturizers than that of the young skin. An age-dependent decrease in ceramides content is well known and correlates with skin dryness and loss of elasticity at an advanced age [12, 24-26].

Our study demonstrates that young skin is not persistently dependent on a moisturizer as demonstrated by a skin recovery time of 6 days in winter and 11 days until reaching the skin's condition at baseline and of 21 days until matching the control side in summer. Surprisingly, the control side for young patients becomes better over the observed time period in the summer study, which explains our two definitions of recovery time above. We assume that this is a bias from the participants as they might see the moisturized side has less skin symptoms over time or they might be less stringent in their evaluation over time.

Age affects skin recovery time. Stopping moisturizer use in aging persons did not result in recovery after 3 weeks, in contrast to use in younger women. We hypothesize that older persons need longer time after stopping moisturizers to recover to their original skin condition. It is unclear if the small difference is noticeable for the participant with aging skin itself and therefore is rather a subjective finding, which could be a result of the nocebo effect. As far as all subjects from the older group were concerned, some were even convinced that by stopping the application of their moisturizer would cause their skin to wrinkle more; it is possible that their negative mindset resulted in a biased perception.

Older female subjects' greatest fear at baseline was an increase in wrinkles, which is a widespread dread of older women [27]. Various studies support the rapid effectiveness of facial creams to reduce the appearance of fine lines and wrinkles [28, 29]. The majority of young participants concluded to stop using moisturizers after participation in our study, as they did not recognize any additional benefit for their facial skin. In contrast, all aging women restarted their moisturizer, which shows that its use is 
often influenced and shaped by subjective perception.

However, on the basis of current data, efficacy and disadvantages of moisturizing treatment depend on the complexity of formulations and the individual skin type $[12,19,30]$. A limitation of our study is the low number of participants with a short follow-up time. A larger cohort, a facial recognition and scanning technology, and a variety of laboratory and in vivo analyses would have provided more statistical power for more reliable conclusions. Another limiting factor for more personalized results was our present study's lack of analysing different skin types. Furthermore, the study design did not allow blinding of subjects because of a conscious discontinuation of a previous treatment, which could have affected the subjects' self-assessments. Therefore, the results should be interpreted with caution. A follow-up study with a skin analysis imaging system based on artificial intelligence as an objective assessment tool and tape stripping to the evaluate skin barrier is planned.

\section{CONCLUSION}

This study demonstrates that both healthy young and aging facial skin react with temporary dryness to a sudden stop of a previous longterm moisturizer application, and can recover fully without moisturizers to the initial state in younger but not older women. Healthy skin renews itself, regenerates and differentiates keratinocytes continuously. The skin recovery time for skin dehydration is 1-3 weeks in female subjects under 25 years of age with varying intensities depending on the season. In older participants the recovery time was not achieved in the 3-week observation period. It cannot be concluded whether the effect was caused by the nocebo effect or not. It is also possible that their skin might need more than 21 days to recover or a reapplication of moisturizer. Further studies based on molecular mechanisms and individual skin types are needed to investigate the effects of long-term moisturizer interruption in healthy individuals.

\section{ACKNOWLEDGEMENTS}

The authors thank the participants of the study.

Funding. No funding or sponsorship was received for this study or publication of this article.

Authorship. All named authors meet the International Committee of Medical Journal Editors (ICMJE) criteria for authorship for this article, take responsibility for the integrity of the work as a whole, and have given their approval for this version to be published.

Authorship Contributions. Alexander Navarini designed the study. Julia-Tatjana Maul and Lara Valeska Maul wrote the manuscript. All authors critically reviewed the manuscript. All authors have read and approved the final manuscript.

Disclosures. Julia-Tatjana Maul is an employee of USZ and holds a "Filling the GAP" scholarship. J-T Maul has served as advisor and/ or received speaking fees and/or participated in clinical trials sponsored by AbbVie, Almirall, Amgen, BMS, Celgene, Eli Lilly, LEO Pharma, Janssen-Cilag, MSD, Novartis, Pfizer, Pierre Fabre, Roche, Sanofi, UCB. Lara Valeska Maul has served as advisor and/or received speaking fees and/or participated in clinical trials sponsored by Amgen, BMS, Celgene, Eli Lilly, MSD, Novartis, Pierre Fabre, Roche, Sanofi. Florian Anzengruber has served as consultant, speaker and advisor for various pharmaceutical companies, including Abbvie, Celgene (Amgen), Leo Pharma, Galderma, Eli Lilly, Almirall, Janssen Cilag, UCB Pharma and Novartis. Martin Kägi: Advisory Boards: Galderma, Novartis, Lilly, Sanofi, Jansen, Abbvie, L'Oréal, Merz, Filabé, Blidor, Biomed. Clinical Studies: Novartis. Ambassador and Key Opinion Leader/Trainer: Merz, Filmed, Allergan, Sinclair, Algeness. Alexander A. Navarini has been a consultant and advisor and/or received speaking fees and/ or grants and/or served as an investigator in clinical trials for the following companies: AbbVie, Almirall, Amgen, BMS, Boehringer Ingelheim, Celgene, Eli Lilly, GSK, LEO Pharma, 
Janssen-Cilag, MSD, Novartis, Pfizer, Sandoz, Sanofi, UCB. A.A. Navarini does not hold any shares or other financial interest in any related pharmaceutical company. Marc Kägi, Phil Cheng, Mathilde von Laue and Yuk Yi Chen have nothing to disclose.

Compliance with Ethics Guidelines. The clinical studies were ascertained by consultation with the Swiss Ethics Committee prior to the start of the study (Ethics Committee Northwest Switzerland EKNZ 2020-00667) and in full accordance with the Declaration of Helsinki (1964) and its subsequent amendments. Good Clinical Practice was maintained throughout the studies. Informed consent was received from the patients for inclusion in the study.

Data Availability. All data generated or analyzed during this study are included in this published article/as supplementary information files.

Open Access. This article is licensed under a Creative Commons Attribution-NonCommercial 4.0 International License, which permits any non-commercial use, sharing, adaptation, distribution and reproduction in any medium or format, as long as you give appropriate credit to the original author(s) and the source, provide a link to the Creative Commons licence, and indicate if changes were made. The images or other third party material in this article are included in the article's Creative Commons licence, unless indicated otherwise in a credit line to the material. If material is not included in the article's Creative Commons licence and your intended use is not permitted by statutory regulation or exceeds the permitted use, you will need to obtain permission directly from the copyright holder. To view a copy of this licence, visit http://creativecommons.org/licenses/by$\mathrm{nc} / 4.0 /$.

\section{REFERENCES}

1. Pons-Guiraud A. Dry skin in dermatology: a complex physiopathology. J Eur Acad Dermatol Venereol. 2007;21(Suppl 2):1-4.

2. Mayo Clinic. Dry skin. Mayo Clinic. 2019. www. mayoclinic.com/health/dry-skin/DS00560. Accessed 4 Aug 2020.

3. Alexander H, Brown S, Danby S, Flohr C. Research techniques made simple: transepidermal water loss measurement as a research tool. J Invest Dermatol. 2018;138(11):2295-2300 e1.

4. Jiang ZX, DeLaCruz J. Appearance benefits of skin moisturization. Skin Res Technol. 2011;17(1):51-5.

5. Loden M. Barrier recovery and influence of irritant stimuli in skin treated with a moisturizing cream. Contact Dermat. 1997;36(5):256-60.

6. Held E, Lund H, Agner T. Effect of different moisturizers on SLS-irritated human skin. Contact Dermat. 2001;44(4):229-34.

7. Buraczewska I, Berne B, Lindberg $M$, Törmä $H$, Lodén M. Changes in skin barrier function following long-term treatment with moisturizers, a randomized controlled trial. $\mathrm{Br} \mathrm{J}$ Dermatol. 2007;156(3):492-8.

8. Serup J. EEMCO guidance for the assessment of dry skin (xerosis) and ichthyosis: clinical scoring systems. Skin Res Technol. 1995;1(3):109-14.

9. MeteoSchweiz. Klimabulletin Winter 2016/2017. MeteoSchweiz. 2017. https://www.meteoschweiz. admin.ch/content/dam/meteoswiss/de/Ungebunde ne-Seiten/Publikationen/Klimabulletin/doc/klimab ulletin_winter_2016-2017-korr.pdf. Accessed 4 Aug 2020 .

10. MeteoSchweiz. Klimabulletin Sommer 2017. MeteoSchweiz. 2017. https://www.meteoschweiz. admin.ch/content/dam/meteoswiss/de/service-und -publikationen/Publikationen/doc/klimabulletin_so mmer_d-2017.pdf. Accessed 4 Aug 2020.

11. Rawlings AV, Canestrari DA, Dobkowski B. Moisturizer technology versus clinical performance. Dermatol Ther. 2004;17(Suppl 1):49-56.

12. Draelos ZD. Modern moisturizer myths, misconceptions, and truths. Cutis. 2013;91(6):308-14.

13. Levin J, Momin SB. How much do we really know about our favorite cosmeceutical ingredients? J Clin Aesthet Dermatol. 2010;3(2):22-41. 
14. Held E, Agner T. Effect of moisturizers on skin susceptibility to irritants. Acta Derm Venereol. 2001;81(2):104-7.

15. Fu JJ, Hillebrand GG, Raleigh P, et al. A randomized, controlled comparative study of the wrinkle reduction benefits of a cosmetic niacinamide/peptide/retinyl propionate product regimen vs a prescription $0.02 \%$ tretinoin product regimen. $\mathrm{Br} \mathrm{J}$ Dermatol. 2010;162(3):647-54.

16. Crowther JM, Sieg A, Blenkiron P, et al. Measuring the effects of topical moisturizers on changes in stratum corneum thickness, water gradients and hydration in vivo. Br J Dermatol. 2008;159(3): 567-77.

17. Heinrich U, Koop U, Leneveu-Duchemin M-C, et al. Multicentre comparison of skin hydration in terms of physical-, physiological- and product-dependent parameters by the capacitive method (Corneometer CM 825). Int J Cosmet Sci. 2003;25(1-2):45-53.

18. Draelos ZD, Matsubara A, Smiles K. The effect of $2 \%$ niacinamide on facial sebum production. J Cosmet Laser Ther. 2006;8(2):96-101.

19. Lee C, Bajor J, Moaddel T, et al. Principles of moisturizer product design. J Drugs Dermatol. 2019;18(1s):s89-95.

20. Guenther L, Lynde CW, Andriessen A, et al. Pathway to dry skin prevention and treatment. J Cutan Med Surg. 2012;16(1):23-31.

21. Rogers J, Harding C, Mayo A, Banks J, Rawlings A. Stratum corneum lipids: the effect of ageing and the seasons. Arch Dermatol Res. 1996;288(12):765-70.

22. Hellemans L, Corstjens H, Neven A, Declercq L, Maes D. Antioxidant enzyme activity in human stratum corneum shows seasonal variation with an age-dependent recovery. J Invest Dermatol. 2003;120(3):434-9.

23. Wei KS, Stella C, Wehmeyer KR, et al. Effects of season stratum corneum barrier function and skin biomarkers. J Cosmet Sci. 2016;67(3):185-203.

24. Liu X, German GK. The effects of barrier disruption and moisturization on the dynamic drying mechanics of human stratum corneum. J Mech Behav Biomed Mater. 2015;49:80-9.

25. White-Chu EF, Reddy M. Dry skin in the elderly: complexities of a common problem. Clin Dermatol. 2011;29(1):37-42.

26. Trayssac M, Hannun YA, Obeid LM. Role of sphingolipids in senescence: implication in aging and age-related diseases. J Clin Invest. 2018;128(7): 2702-12.

27. Forte AJ, Andrew TW, Colasante C, Persing JA. Perception of age, attractiveness, and tiredness after isolated and combined facial subunit aging. Aesthetic Plast Surg. 2015;39(6):856-69.

28. Pinsky MA. Efficacy and safety of an anti-aging technology for the treatment of facial wrinkles and skin moisturization. J Clin Aesthet Dermatol. 2017;10(12):27-35.

29. Spada F, Lui AH, Barnes TM. Use of formulations for sensitive skin improves the visible signs of aging, including wrinkle size and elasticity. Clin Cosmet Investig Dermatol. 2019;12:415-25.

30. Mukta S, Adam F. Cosmeceuticals in day-to-day clinical practice. J Drugs Dermatol. 2010;9(5 Suppl ODAC Conf Pt 1):s62-s6666. 